\title{
Lovastatin Inhibits EMT and Metastasis of Triple- Negative Breast Cancer Stem Cells Through Dysregulation of Cytoskeleton-Associated Proteins
}

\section{Chanjuan Zheng}

Hunan Normal University

\section{Shichao Yan}

Hunan Normal University

Lu Lu

Hunan Normal University

Hui Yao

Hunan Normal University

\section{Guangchun He}

Hunan Normal University

\section{Sisi Chen}

Hunan Normal University

\section{Ying Li}

Hunan Normal University

\section{Xiaojun Peng}

Jingjie PTM Biolab Co. Ltd

Zhongyi Cheng

Jingjie PTM BioLab Co. Ltd

Mi Wu

Hunan Normal University

\section{Qiuting Zhang}

Hunan Normal University

\section{Guifei Li}

Hunan Normal University

\section{Shujun Fu}

Hunan Normal University

Xiyun Deng ( $\nabla$ dengxiyunmed@hunnu.edu.cn )

Hunan Normal University https://orcid.org/0000-0003-2203-970X 
Keywords: Lovastatin, Triple-negative breast cancer, Cancer stem cells, Epithelial-to-mesenchymal transition, Cytoskeleton

Posted Date: January 19th, 2021

DOI: https://doi.org/10.21203/rs.3.rs-147845/v1

License: (1) This work is licensed under a Creative Commons Attribution 4.0 International License. Read Full License 


\section{Abstract \\ Background}

Triple-negative breast cancer (TNBC) is more aggressive and has poorer prognosis compared to other subtypes of breast cancer. Epithelial-to-mesenchymal transition (EMT) is a process in which epithelial cells transform into mesenchymal-like cells capable of migration, invasion, and metastasis. Recently, we have demonstrated that lovastatin, a 3-hydroxy-3-methylglutaryl-coenzyme A reductase inhibitor and a lipid-lowering drug, could inhibit stemness properties of cancer stem cells (CSCs) derived from TNBC cell in vitro and in vivo. This study is aimed at investigating whether lovastatin inhibits TNBC CSCs by inhibiting EMT and suppressing metastasis and the mechanism involved.

\section{Methods}

LC-MS/MS was used to identify differentially regulated lysine acylation between TNBC and non-TNBC CSCs. In vivo, two nude mouse models were used to study the tumor growth, EMT phenotype, and metastasis of breast CSCs. The effect of lovastatin on EMT-related proteins was examined by immunohistochemistry, western blot and immunofluorescence-confocal microscopy. TGF- $\beta 1$-challenged immortalized mammary epithelial cells MCF10A were treated with lovastatin and the change of cell morphology were detected by microscopic examination. Cell migration was detected by wound-healing assay. The formation of pseudopodia and the distribution of F-actin were investigated by transmission electron microscopy (TEM) and immunofluorescence-confocal microscopy, respectively. Bioinformatics analysis was used to evaluate the correlation between the expression of cytoskeleton-associated genes and overall survival of breast cancer patients.

\section{Results}

Lovastatin dysregulated lysine succinylation of cytoskeleton-associated proteins in CSCs derived from TNBC MDA-MB-231 cell. Lovastatin inhibited EMT as demonstrated by down-regulation of the protein levels of Vimentin and Twist in MDA-MB-231 CSCs in vitro and vivo and by reversal of TGF- $\beta 1$-induced morphological change in MCF10A cells. Combination of lovastatin with doxorubicin synergistically inhibited liver metastasis of MDA-MB-231 CSCs. Lovastatin also inhibited the migration of MDA-MB-231 CSCs. The disruption of cytoskeleton in TNBC CSCs by lovastatin was demonstrated by the reduction of the number of pseudopodia and the relocation of F-actin cytoskeleton. Bioinformatics analysis revealed that higher expression levels of cytoskeleton-associated genes were characteristic of TNBC and predicted poorer overall survival in breast cancer patients.

\section{Conclusions}


Lovastatin could inhibit the EMT and metastasis of TNBC CSCs in vitro and in vivo through dysregulation of cytoskeleton-associated proteins.

\section{Background}

Breast cancer is by far the most common malignancy and the second leading cause of cancer-related mortality among women (1). It is a type of heterogeneous disease that differs in pathomorphology, biology, clinical manifestation, and treatment response (2). Triple-negative breast cancer (TNBC), as a subtype of breast cancer, accounts for $10-20 \%$ of them. Owing to the lack of expression of estrogen receptor (ER), progesterone receptor (PR) and lack of expression or amplification of human epidermal growth factor receptor 2 (HER2), TNBC patients are insensitive to endocrine therapy or molecular targeted therapy, resulting in high recurrence and metastatic potential (3). Currently, new treatment strategies for TNBC, including poly (ADP-ribose) polymerase (PARP) inhibition and immune checkpoint inhibition, are being actively developed in preclinical and clinical studies $(4,5)$. Unfortunately, only a small proportion of TNBC patients could benefit from these treatments $(6,7)$. Therefore, it is an urgent task to explore promising targeted drugs so as to improve the efficacy for TNBC.

There is substantial evidence that breast cancer development is hierarchically organized and driven by a minute population of cancer cells known as cancer stem cells (CSCs) which contribute to tumor metastasis and relapse $(8,9)$. Targeting CSCs has become a popular goal for treating a wide range of tumor types, and may be especially important for TNBC patients (10). Numerous clinical trials have been conducted for targeting breast cancer CSCs (11), but limited data currently exist clinically for the treatment of TNBC (10). Therefore, discovery of drugs that target CSCs will have an enormous impact in TNBC therapeutics. In the process of cancer metastasis, CSCs undergo epithelial-to-mesenchymal transition (EMT), thereby acquiring mesenchymal features which have the ability to migrate and invade $(12,13)$. EMT involves the loss of intracellular cohesion, disruption of the extracellular matrix (ECM), modifications of the cytoskeleton, and increased cell motility and invasiveness $(14,15)$. Accumulating evidence showed that EMT-inducible factors also enhance or induce CSC-like features in cancer cells. Within breast cancer, the acquisition of tumor stem cell-like features, the formation of tumor spheres and the appearance of a breast cancer stem cell-specific phenotype $\left(\mathrm{CD} 44^{+} / \mathrm{CD} 24^{-}\right)$were all promoted by the occurrence of EMT (16).

Lovastatin is a natural statin derived from Monascus-fermented rice or dioscorea and occurs at a high content in Oystermushroom (17). It has been widely used in prevention and treatment of hyperlipidemia (18). In the last two decades, the antitumor effect of lovastatin has gained increasing attention (19). In vitro studies have shown that lovastatin could inhibit the cell cycle progression (20), induce apoptosis (21), and suppress cell migration and invasion (22). In vivo, lovastatin could suppress the growth of transplanted tumor or prevent pulmonary metastasis derived from breast cancer (20). Recently, we have demonstrated that lovastatin could inhibit stemness properties of CSCs derived from TNBC cell lines in vitro, in a mouse model of orthotopic tumor growth, and in a patient-derived xenograft (PDX) model ( 
(23-25) and manuscript in preparation). Our findings support the evidence that lovastatin may be a candidate drug for the treatment of TNBC.

Post translational modifications (PTMs) are one of the most efficient biological mechanisms for expanding the genetic code and for regulating cellular pathophysiology. Lysine succinylation (Ksucc), a newly identified form of PTMs, could cause significant changes in the structure and function of proteins (26). Several lines of evidence suggest that Ksucc has been involved in the initiation and development of numerous different types of tumors, such as gastric and breast cancer (27-29). However, Ksucc also exerts tumor-inhibitory effect in hepatocellular carcinoma and intestinal cancer $(30,31)$. Importantly, the mechanism of some anti-tumor drugs may also be related to Ksucc modification. For example, heat shock protein 90 (HSP90) inhibitor exhibits an anti-tumor activity against bladder cancer by affecting Ksucc modification (32).

Through global profiling of lysine acylation, we found that lovastatin preferentially targets CSCs derived from TNBC over non-TNBC cells through Ksucc of proteins involved in cytoskeleton. Our studies demonstrated that lovastatin could selectively inhibit the viability of TNBC CSCs in vitro and in vivo. Therefore, this study aims to further investigate whether lovastatin exerts its anticancer effect in TNBC CSCs through inhibiting the EMT program and metastasis via regulation of cytoskeleton-associated proteins.

\section{Materials And Methods Key Reagents}

Lovastatin (ab120614) was obtained from Abcam (Cambridge, UK) and dissolved in DMSO at a stock concentration of 20 or $30 \mathrm{mM}$ and stored at $-80^{\circ} \mathrm{C}$ before use. Doxorubicin was purchased from Selleck, dissolved in DMSO and stored as directed. Human recombinant TGF- $\beta 1$ was purchased from R\&D Systems (Minneapolis, MN) and was dissolved in an aqueous solvent (vehicle) containing $4 \mathrm{mM} \mathrm{HCl}$ and $1 \mathrm{mg} / \mathrm{ml} \mathrm{BSA}$.

\section{Cell lines}

Breast cancer cell lines MDA-MB-231 (TNBC) and MDA-MB-453 (non-TNBC) were purchased from the Cell Resource Center of Shanghai Institutes for Biological Sciences, maintained in DMEM medium supplemented with $10 \%$ fetal bovine serum. The immortalized mammary epithelial cell line MCF10A was obtained from Kunming Institute of Zoology, Chinese Academy of Sciences. MCF10A cells were maintained in DMEM/F-12 medium supplemented with $5 \%$ horse serum, $20 \mathrm{ng} / \mathrm{mL} E G F, 500 \mathrm{ng} / \mathrm{mL}$ hydrocortisone, $100 \mathrm{ng} / \mathrm{mL}$ cholera toxin and $10 \mu \mathrm{g} / \mathrm{mL}$ insulin. All cell lines were routinely cultured at $37^{\circ} \mathrm{C}$ with $21 \% \mathrm{O}_{2}$ and $5 \% \mathrm{CO}_{2}$ and were tested negative for mycoplasma contamination. 


\section{Enrichment and characterization of breast cancer stem cells}

MDA-MB-231 or MDA-MB-453 cells were trypsinized to single cells and seeded into 6-well ultra-low attachment plates $(2,500$ cells $/ \mathrm{mL})$ using breast cancer stem cell medium (DMEM/F12, $1 \times$ B27, 20 $\mathrm{ng} / \mathrm{mL} E G F, 20 \mathrm{ng} / \mathrm{mL} \mathrm{bFGF}, 0.4 \% \mathrm{BSA}, 4 \mu \mathrm{g} / \mathrm{mL}$ insulin and $0.2 \%$ hydrocortisone) (33). CD $44^{+} / \mathrm{CD} 24^{-}$ cells were sorted by sequential magnetic sorting after addition of beads coated with anti-CD44 or antiCD24 antibody. These cells with CSC-like properties were designated sphere-forming cells (SFCs) to distinguish from their parental cells (PCs). The CSC phenotype was characterized by prolonged mammosphere formation in ultra-low attachment culture and by their enhanced tumorigenic ability as demonstrated by two orders of magnitude higher tumorigenicity of SFCs than PCs in nude mice (Additional file 1: Figure S1, Additional file 2: Table S1).

\section{Immunofluorescence - laser scanning confocal microscopy}

The cover glasses were put into 6-cm dishes, and MDA-MB-231 CSCs $\left(1 \times 10^{5}\right.$ cells $\left./ \mathrm{mL}\right)$ were seeded and allowed to grow overnight at $37^{\circ} \mathrm{C}$. The cells were treated with different concentrations of lovastatin for 48 hours. These cover glasses were fixed with $4 \%$ paraformaldehyde and collected for indirect immunofluorescence staining. The primary antibodies included those against Vimentin (ZSGB-BIO, Cat\#ZM0260, Mouse, 1:100) or Twist (Abcam, Cat\#ab50581, Rabbit, 1:100). The secondary antibodies were DyLight 488 anti-mouse IgG $(\mathrm{H}+\mathrm{L})$ (Vector, Cat\#DI-2488, 1:100) and DyLight 594 anti-rabbit IgG $(\mathrm{H}+\mathrm{L})$ (Vector, Cat\#DI-1594, 1:100), respectively.

\section{Western blot analysis}

Cultured cells were lysed using $1 \times$ cell lysis buffer (Cell Signaling Technology, Danvers, MA, USA) with 1× protease inhibitor cocktail (Complete Mini, Roche, Mannheim, Germany) and $1 \mathrm{mM}$

phenylmethanesulfonyl fluoride (Sigma-Aldrich) added. After centrifugation, the supernatants (whole cell lysates) were collected and quantified by the BCA protein quantification method. The lysates were mixed with the LDS sample buffer and reducing agent and denatured by boiling. The same quantity of protein from each sample was then separated on 10\% denaturing PAGE gels followed by incubation with the respective primary antibodies (Vimentin, CST, Cat\#5741, 1:1,000; Twist, Abcam, Cat\#ab50581, 1:1,000; GAPDH, ZSGB-BIO, Cat\#TA08, 1:10,000) and the HRP-conjugated secondary antibody followed by subsequent ECL development according to our standard procedure (34).

\section{Nude mouse models}


Balb/c-nu mice (female, 5 - 6 weeks old, weight 16 - $18 \mathrm{~g}$ ) were purchased from Hunan SJA Laboratory Animals Co., Ltd. The mice were maintained on a regular sterile diet under SPF animal house conditions. For the model of orthotopic tumor growth and EMT phenotype (Fig. 2a), CSCs resuspended in cold 1x PBS were injected into the fourth mammary fat pad. Two weeks later, the nude mice were randomly grouped based on tumor sizes ( $n=10 /$ group). Lovastatin $(2 \mathrm{mg} / \mathrm{kg}$ ) or vehicle (PBS) was administered twice weekly through oral gavage until the end of the experiment. The tumor growth was monitored by measuring the major $(a)$ and minor $(b)$ axes of the tumor using a caliper twice weekly. The tumor volume $(V)$ was estimated by the equation $V=\left(a \times b^{2}\right) / 2$ as described (35). Three weeks after drug treatment, the mice were sacrificed and the tumors were resected, weighed, and photographed. Part of the tumor tissue was fixed in $4 \%$ buffered formaldehyde and subjected to routine paraffin-embedding and microtome sectioning.

A model of tumor metastasis (Fig. 5a) was generated by injecting the CSCs $\left(5 \times 10^{3} / 100 \mu \mathrm{L} /\right.$ animal) into the tail vein of the nude mice. The mice were randomized into the following four groups ( $n=8 /$ group): saline control, doxorubicin (1 mg/kg), lovastatin (2 mg/kg), and doxorubicin (1 mg/ $\mathrm{kg})+$ lovastatin (2 $\mathrm{mg} / \mathrm{kg}$ ). Drug administration started the next day after tumor cell injection and continued twice weekly for 7 weeks. At the end of drug treatment, the mice were sacrificed and the tumors and the livers resected, weighed, and photographed. Part of the tumor tissue was fixed in $4 \%$ buffered formaldehyde and subjected to routine paraffin-embedding, microtome sectioning, and H\&E or immunohistochemical staining. Metastatic burden was evaluated by counting the metastatic nodules on the surface of each liver. All animal studies were approved by the Hunan Normal University Animal Care Committee.

\section{Immunohistochemistry}

Immunohistochemical staining was carried out using the PV-9000 plus poly-HRP anti-mouse/rabbit IgG detection system as described in our previous study (36). The details of primary antibodies were as follows: Vimentin, CST, Cat\#5741, 1:100; Twist, Abcam, Cat\#ab50581, 1:100. After immunohistochemical staining, the tissue sections were scanned using Automated Quantitative Pathology Imaging System (Vectra, PerkinElmer, Hopkinton, MA, USA) and the total intensity score (TIS) was calculated each from six randomly chosen images at $40 \times$ magnification.

\section{Wounding healing assay}

MDA-MB-231 or MDA-MB-453 CSCs were seeded at a density of $2 \times 10^{5}$ cells/well in 6-well plates. When the cells have grown and fused to $80 \%$, the tip head was used to scratch the central area of the plate well. Lovastatin $(1 \mu \mathrm{M})$ was added to the cells cultured in DMEM medium supplemented with $3 \%$ fetal bovine serum. The cell migration distance in the scratch area was measured at 0 and 24 hours, respectively.

\section{LC-MS/MS analysis and data search}


The CSCs were treated with lovastatin $(1 \mu \mathrm{M})$ or vehicle in stem cell medium at $37^{\circ} \mathrm{C}$ for $48 \mathrm{~h}$. The cells were then collected by centrifugation, washed with PBS, and snap-frozen in liquid nitrogen, followed by protein extraction and trypsin digestion. The resulting peptides were labeled with tandem mass tag (TMT) isobaric reagents and fractionated by strong cation exchange chromatography. Succinylated peptides were immunoprecipitated with pan-Ksucc antibody-conjugated beads. Enriched peptides were analyzed by liquid chromatography coupled to an Orbitrap Q Exactive ${ }^{\mathrm{TM}}$ Plus. Non-enriched peptides (for proteomics) were fractionated by high pH reverse-phase HPLC using the Agilent 300 Extend $\mathrm{C} 18$ column followed by LC-MS/MS analysis. The resulting MS/MS data was processed using MaxQuant with integrated Andromeda search engine (v.1.5.2.8). Tandem mass spectra were searched against Swissprot human database concatenated with reverse decoy database. False discovery rate (FDR) thresholds for protein, peptide, and modification site were specified at $1 \%$. Minimum peptide length was set at 7 . For quantification method, TMT 6-plex was selected. The site localization probability was set at $\geq 0.75$. The relative changes of Ksucc-modified proteins were normalized to the respective protein level revealed by global proteomic profiling.

\section{Bioinformatics analyses of gene expression levels and breast cancer patient survival}

To explore the expression levels of cytoskeleton-related genes in TNBC and non-TNBC, we analyzed the RNA-seq data of 115 TNBC and 982 non-TNBC clinical samples from the cBioPortal database (http://www.cbioportal.org/datasets). After obtaining the results, we plotted the value of the ordinate to the gene expression converted by log10. We then integrated four datasets, i.e., GSE42568, Nathan Kline Institute (NKI), and GSE3494-U133A, and GSE1456-GPL97 to analyze the overall survival of breast cancer patients between high and low expression levels of cytoskeleton-related genes. Results were obtained with the PROGgeneV2 tool (http://genomics.jefferson.edu/proggene/).

\section{Transmission electron microscopy}

To investigate the formation of pseudopodia, the CSCs were treated with lovastatin $(1 \mu \mathrm{M})$ or vehicle for $48 \mathrm{~h}$, fixed with $2.5 \%$ glutaraldehyde, and post-fixed in $1 \%$ osmium tetroxide (OsO4) for $1-2 \mathrm{~h}$ at $4{ }^{\circ} \mathrm{C}$. The samples were then dehydrated in a graded series of acetone $(50 \%, 70 \%, 90 \%$, and $100 \%)$ and embedded in Epon-Araldite resin. Ultra-thin sections $(50-100 \mathrm{~nm})$ were cut using the ultramicrotome and stained with $3 \%$ uranyl acetate and lead nitrate. The cell morphology was observed and the images acquired using an HT7700 Transmission Electron Microscope (Hitachi, Tokyo, Japan).

\section{Statistical analysis}

All the quantitative data were presented as mean \pm SEM. Statistical analyses (ANOVA, unpaired Student's $t$ test) were carried out using SigmaPlot (version 12.5). $P<0.05$ was considered as statistically 
significant. $\mathrm{IC}_{50}$ was calculated using the GraphPad Prism 5 software. Drug interaction between lovastatin and doxorubicin was assessed using the CompuSyn software to calculate the combination index $(C l)$, with $C l<1, C l=1$, and $C l>1$ indicating synergistic, additive, and antagonistic actions, respectively.

\section{Results}

\section{Lovastatin dysregulates lysine succinylation of cytoskeleton-associated proteins}

Lysine acylations, novel forms of post-translational modifications, play a key role in drug-induced cytotoxicity (37). To investigate the pathways of targeted by lovastatin in TNBC CSCs, we compared lysine acylations of TNBC cells (MDA-MB-231 CSCs) with non-TNBC cells (MDA-MB-453 CSCs). We found that lysine succinylation (Ksucc) was a major lysine acylation type dysregulated by lovastatin in MDAMB-231 CSCs compared with MDA-MB-453 CSCs (Additional file 3: Figure S2). Bioinformatics analyses were performed to annotate the proteins differentially modified by Ksucc in response to lovastatin treatment. Gene Ontology (GO) analysis showed that Ksucc-modified proteins were mainly involved in cytoskeleton organization (such as actin binding) (Fig. 1a). Protein-protein interaction network analysis based on Search Tool for the Retrieval of Interacting Genes (STRING) (http://string-db.org/) showed the proteins (FLNA, TMSB10, STMN, TPM3, MSN, SPTAN1, DSTN, and EZR) of cytoskeleton organization as key mediators of lovastatin's action in MDA-MB-231 CSCs (Fig. 1b). Subcellular localization analysis revealed that most of the succinylated proteins were distributed in the cytoplasm and the nucleus (Fig. 1c), consistent with their localization and role in regulating the cytoskeleton organization.

\section{Lovastatin inhibits epithelial-to-mesenchymal transition of TNBC CSCs}

The orthotopic xenograft model of mammary fat pad injection was used to study the tumor growth and EMT phenotype (Fig. 2a). Each of the tumors was spherical or irregular in shape and gray or gray-red in color. For the mice receiving MDA-MB-231 CSCs, the average tumor volume of the lovastatin-treated group was smaller than that of the control group $(P<0.05)$. For the mice receiving MDA-MB-453 CSCs, the average tumor volume of the lovastatin-treated group was even larger compared with the control group $(P<0.05)$ (Additional file 4:Figure S3a). Tumor weight analysis at the end of the experiment confirmed the results of tumor volume measurement (Additional file 4:Figure S3b). Immunohistochemical staining was performed to evaluate the EMT-related proteins on the orthotopic tumors. We found that in xenograft tumors derived from MDA-MB-231 CSCs, the lovastatin-treated group had a lower score of the mesenchymal markers Vimentin and Twist than the control group $(P<0.05)$. Again, in MDA-MB-453 CSCs tumors, there was no statistical difference in Vimentin and Twist between the lovastatin-treated group and the control group (Fig. 2b). 
MDA-MB-231 and MDA-MB-453 CSCs were treated with different concentrations of lovastatin (0.3 - 3 $\mu \mathrm{M})$ for $48 \mathrm{~h}$. Western blot analysis revealed that the protein levels of Vimentin and Twist were decreased by lovastatin treatment in a dose-dependent manner in MDA-MB-231 CSCs, but the opposite results were obtained with MDA-MB-453 CSCs (Fig. 3a). The expression of Vimentin and Twist was further investigated by immunofluorescence and laser scanning confocal microscopy. The fluorescence intensity of Vimentin (green) and Twist (red) was decreased by lovastatin treatment in MDA-MB-231 but not MDAMB-453 CSCs (Fig. 3b).

The effect of lovastatin on EMT was further investigated by another cell model of induced EMT. Addition of TGF- $\beta 1$ to immortalized epithelial cells such as MCF10A is a well-recognized cell model of inducing EMT in vitro (38). When treated with TGF- $\beta 1$ (160 pM), MCF10A cells could form the loose linked spindle morphology. Addition of lovastatin $(0.3-1 \mu \mathrm{M})$ reversed the change of cell morphology induced by TGF$\beta 1$ (Fig. 3c). Western blot analysis revealed that lovastatin treatment reversed the change of the protein levels of EMT markers induced by TGF- $\beta 1$ (data not shown). These results demonstrate the inhibitory effect of lovastatin on the EMT of TNBC CSCs.

\section{Lovastatin promotes chemosensitization and inhibits metastasis of TNBC CSCs}

Since CSCs contribute to chemoresistance (39), we next investigated whether lovastatin synergizes with the standard chemotherapeutic drug to elicit greater inhibitory effect. We demonstrated that lovastatin sensitizes MDA-MB-231 CSCs to doxorubicin, a standard chemotherapeutic drug for breast cancer therapy. Confocal microscopy of autofluorescence revealed that lovastatin promoted intracellular accumulation of doxorubicin in MDA-MB-231 CSCs (Fig. 4a). Furthermore, lovastatin synergized with doxorubicin to inhibit tumorsphere formation of MDA-MB-231 CSCs (Fig. 4b).

Another nude mouse model of tail vein injection (Fig. 5a) was further used to evaluate the synergistic effect of combination treatment on metastasis of TNBC CSCs to distal organs. We found that doxorubicin alone had no inhibition and lovastatin alone showed $46.2 \pm 21.7 \%$ inhibition on liver metastasis of MDA-MB-231 CSCs. However, combination of lovastatin with doxorubicin synergistically inhibited the majority of liver metastasis of MDA-MB-231 CSCs as demonstrated by a $81.5 \pm 5.8 \%$ reduction of the macroscopic nodules (Fig. 5b). Histopathological examination confirmed the synergistically inhibitory effect on cancer cell colonization in the liver of the combination treatment group (Fig. 5c).

In addition, wounding healing assay was used to evaluate the effect of lovastatin on TNBC CSCs migration. CSCs were treated with lovastatin and photographed at 0 and $24 \mathrm{~h}$ respectively after cell scratching. We found that the migration area of the lovastatin-treated group was significantly larger than that of the vehicle-treated group in MDA-MB-231 CSCs. However, there was no obvious inhibitory effect on migration in MDA-MB-453 CSCs (Fig. 6a). 
Considering the formation of pseudopodia is supported by actin cytoskeleton, we evaluated whether lovastatin caused disruption of pseudopodia in TNBC cells. As expected, transmission electron microscopy (TEM) revealed that lovastatin reduced the number of pseudopodia in MDA-MB-231 but not MDA-MB-453 CSCs (Fig. 6b). We then examined the effect of lovastatin on cytoskeleton by immunofluorescence-confocal microscopic examination of F-actin. Interestingly, we found F-actin seemed to be changed from diffuse distribution in the cytoplasm in untreated cells to nuclear or perinuclear localization in lovastatin-treated MDA-MB-231 CSCs (Fig. 6c). Thus, these data suggest that lovastatin could inhibit liver metastasis and cause disruption of the cytoskeleton in TNBC CSCs.

\section{Higher expression levels of cytoskeleton-associated genes are characteristic of TNBC and predict poorer overall survival in breast cancer patients}

In order to explore how our results might be relevant to the clinic, we compared the expression levels of cytoskeleton-related genes between TNBC and non-TNBC and investigated their associations with breast cancer patient survival. We analyzed the RNA-seq data of TNBC and non-TNBC samples from The Cancer Genome Atlas (TCGA) database for the cytoskeleton-related proteins revealed in Fig. 1b. We found that five out of the eight cytoskeleton-related genes, i.e., FLNA, TMSB10, STMN1, MSN, and TPM3, were expressed at significantly higher levels in TNBC compared with non-TNBC (Fig. 7a-e). Consistent with the RNA-seq data, survival analysis showed that breast cancer patients who had higher levels of these cytoskeleton-related genes had poorer overall survival compared with those with lower levels (Fig. 7f-j). The clinical data suggest that these cytoskeleton-related genes might be potential targets for the treatment of TNBC.

\section{Discussion}

It is well known that EMT and metastasis play an important role in the acquisition of the malignant phenotype of cancer cells (40). We set out to explore whether lovastatin could inhibit breast CSCs by reversal of the EMT program and inhibition of metastasis in TNBC. Our study demonstrated that both the expression of mesenchymal markers such as Vimentin and EMT-related transcription factors such as Twist could be down-regulated by treatment with lovastatin in MDA-MB-231 CSCs. Furthermore, EMT induced by TGF- $\beta 1$ in immortalized mammary epithelial cells MCF10A could also be reversed by lovastatin. The present study also showed that lovastatin could inhibit liver metastasis as evidenced by reduced nodule formation and confirmed histopathologically by eliminated cancer cell colonization. Therefore, our study provided solid evidence that lovastatin could inhibit the EMT program and metastasis in TNBC CSCs in vitro and in vivo.

In the process of EMT, tumor cells gain migratory and metastatic properties that involve a dramatic reorganization of the actin cytoskeleton and the concomitant formation of membrane protrusions required for invasive growth $(40,41)$. Emerging evidence suggests that cytoskeleton regulatory proteins 
are a convergent node of signaling pathways emanating from extracellular stimulus to cell movement. The coordinated activity of various cytoskeleton-binding proteins regulates a variety of cytoskeletonbased processes, including assembly of the microfilament and cell motility.

Actin cytoskeleton remodeling is an upstream regulator of EMT in metastatic breast cancer cells (42), and several studies clarified EMT was driven by actin cytoskeleton remodeling in hepatocellular and colorectal carcinoma $(43,44)$. F-actin cytoskeleton is regulated by various actin-binding proteins, one family of which are the filamins, with filamin A (FLNA), also called actin-binding protein 280 (ABP-280), being the most powerful actin-binding protein, together with actin microfilaments, direct the cell's elasticity and movement $(45,46)$. In our study, we demonstrated lovastatin induced rearrangement of the actin cytoskeleton favoring perinuclear and nuclear localization of F-actin filaments. Location of actin filaments underneath the plasma membrane is important for the formation of cellular protrusions such as lamellipodia and filopodia (47). Our results further showed that the number of pseudopodia of TNBC CSCs after lovastatin-treated were reduced, which confirmed cytoskeleton organization pathway play an important role in the lovastatin inhibition EMT and metastasis of TNBC CSCs. We have demonstrated that lovastatin inhibited the EMT and metastasis of TNBC CSCs. Therefore, it's not surprising that lovastatin modulates these malignant behaviors of TNBC CSCs through dysregulation of cytoskeletonassociated proteins. This is supported by bioinformatics analysis showing that the cytoskeletonassociated genes are differentially expressed between TNBC and non-TNBC tissues samples and that higher expression levels of these genes are associated with poorer prognosis in breast cancer patients.

\section{Conclusions}

In summary, our present study has provided evidence, for the first time, that lovastatin, a natural HMGCoA reductase inhibitor, inhibits TNBC CSCs in vitro and in vivo through inhibition of EMT phenotype and suppression of metastasis by dysregulation of cytoskeleton-associated proteins. This study lays the foundation for the understanding of the inhibitory effect of lovastatin on the EMT and metastasis of TNBC CSCs and has potential clinical implications for the future management of TNBC. Further studies are required to move forward our effort toward resolving the issues of how lovastatin causes disturbance of the cytoskeleton organization pathway and how protein Ksucc contributes to lovastatin-induced EMT and metastasis in TNBC CSCs.

\section{Abbreviations}

Cl: Combination index; CSCs: Cancer stem cells; ECM: Extracellular matrix; EMT: Epithelial-tomesenchymal transition; ER: Estrogen receptor; FDR: False discovery rate; GO: Gene ontology; HER2: Human epidermal growth factor receptor 2; HSP90: heat shock protein 90; Ksucc: Lysine succinylation; PARP: Poly (ADP-ribose) polymerase; PDX: Patient-derived xenograft; PTMs: Post translational modifications; PR: Progesterone receptor; SEM: Standard error of the mean; TCGA: The cancer genome atlas; TEM: transmission electron microscopy; TMT: tandem mass tag; TNBC: Triple-negative breast cancer. 


\section{Declarations}

Acknowledgements

Not applicable.

\section{Authors' contributions}

Conception and design of the research: XYD; acquisition of data: CJZ, SCY, MW囚QTZ, XJP and LL; statistical analysis: CJZ, SSC, ZYC and YL; drafting the manuscript: CJZヌSCY and LL; reviewing the manuscript: GFL, SJF and GCH. All authors have read and approved the final submitted manuscript.

\section{Funding}

This work was supported by the National Natural Science Foundation of China $(81872167,81472496)$, the Natural Science Foundation of Hunan (2019JJ40193), and the Key Project of Department of Education of Hunan Province (14A089).

\section{Availability of data and materials}

Please contact author for data requests.

\section{Ethics approval and consent to participate}

All protocols for the mouse model were approved by the Hunan Normal University Institutional Animal Care and Ethics Committee and carried out in accordance with the guidelines stated in the International Guiding Principles for Biomedical Research Involving Animals.

\section{Consent for publication}

Not applicable.

\section{Competing interests}

We have no competing interests.

\section{References}

1. Siegel RL, Miller KD, Jemal A. Cancer statistics, 2020. CA Cancer J Clin. 2020;70:7-30.

2. Sorlie T, Perou CM, Tibshirani R, Aas T, Geisler S, Johnsen H, et al. Gene expression patterns of breast carcinomas distinguish tumor subclasses with clinical implications. Proc Natl Acad Sci U S A. 2001;98:10869-74.

3. Dent R, Trudeau M, Pritchard KI, Hanna WM, Kahn HK, Sawka CA, et al. Triple-negative breast cancer: clinical features and patterns of recurrence. Clin Cancer Res. 2007;13:4429-34. 
4. Fremd $C$, Jaeger D, Schneeweiss A. Targeted and immuno-biology driven treatment strategies for triple-negative breast cancer: current knowledge and future perspectives. Expert Rev Anticancer Ther. 2019;19:29-42.

5. Lee A, Djamgoz MBA. Triple negative breast cancer: Emerging therapeutic modalities and novel combination therapies. Cancer Treat Rev. 2018;62:110-22.

6. Papadimitriou M, Mountzios G, Papadimitriou CA. The role of PARP inhibition in triple-negative breast cancer: Unraveling the wide spectrum of synthetic lethality. Cancer Treat Rev. 2018;67:34-44.

7. Emens LA, Cruz C, Eder JP, Braiteh F, Chung C, Tolaney SM, et al. Long-term Clinical Outcomes and Biomarker Analyses of Atezolizumab Therapy for Patients With Metastatic Triple-Negative Breast Cancer: A Phase 1 Study. Jama Oncol. 2019;5:74-82.

8. Liu S, Wicha MS. Targeting breast cancer stem cells. J Clin Oncol. 2010;28:4006-12.

9. Islam F, Qiao B, Smith RA, Gopalan V, Lam AK. Cancer stem cell: fundamental experimental pathological concepts and updates. Exp Mol Pathol. 2015;98:184-91.

10. O'Conor CJ, Chen T, Gonzalez I, Cao D, Peng Y. Cancer stem cells in triple-negative breast cancer: a potential target and prognostic marker. Biomark Med. 2018;12:813-20.

11. Dey P, Rathod M, De A. Targeting stem cells in the realm of drug-resistant breast cancer. Breast Cancer (Dove Med Press). 2019;11:115-35.

12. Ishiwata T. Cancer stem cells and epithelial-mesenchymal transition: Novel therapeutic targets for cancer. Pathol Int. 2016;66:601-8.

13. Sato R, Semba T, Saya H, Arima Y. Concise Review: Stem Cells and Epithelial-Mesenchymal Transition in Cancer: Biological Implications and Therapeutic Targets. Stem Cells. 2016;34:19972007.

14. Voulgari A, Pintzas A. Epithelial-mesenchymal transition in cancer metastasis: mechanisms, markers and strategies to overcome drug resistance in the clinic. Biochim Biophys Acta. 2009;1796:75-90.

15. Kraljevic Pavelic S, Sedic M, Bosnjak H, Spaventi S, Pavelic K. Metastasis: new perspectives on an old problem. Mol Cancer. 2011;10:22.

16. May CD, Sphyris N, Evans KW, Werden SJ, Guo W, Mani SA. Epithelial-mesenchymal transition and cancer stem cells: a dangerously dynamic duo in breast cancer progression. Breast Cancer Res. 2011;13:202.

17. Shi YC, Pan TM. Beneficial effects of Monascus purpureus NTU 568-fermented products: a review. Appl Microbiol Biotechnol. 2011;90:1207-17.

18. Le NA. Hyperlipidaemia and cardiovascular disease. Curr Opin Lipidol. 2001;12:587-9.

19. Graaf MR, Richel DJ, van Noorden CJ, Guchelaar HJ. Effects of statins and farnesyltransferase inhibitors on the development and progression of cancer. Cancer Treat Rev. 2004;30:609-41.

20. Yu X, Luo Y, Zhou Y, Zhang Q, Wang J, Wei N, et al. BRCA1 overexpression sensitizes cancer cells to lovastatin via regulation of cyclin D1-CDK4-p21WAF1/CIP1 pathway: analyses using a breast cancer cell line and tumoral xenograft model. Int J Oncol. 2008;33:555-63. 
21. Campbell MJ, Esserman LJ, Zhou Y, Shoemaker M, Lobo M, Borman E, et al. Breast cancer growth prevention by statins. Cancer Res. 2006;66:8707-14.

22. Kang S, Kim ES, Moon A. Simvastatin and lovastatin inhibit breast cell invasion induced by H-Ras. Oncol Rep. 2009;21:1317-22.

23. Song L, Tao X, Lin L, Chen C, Yao H, He G, et al. Cerasomal Lovastatin Nanohybrids for Efficient Inhibition of Triple-Negative Breast Cancer Stem Cells To Improve Therapeutic Efficacy. ACS Appl Mater Interfaces. 2018;10:7022-30.

24. Yang T, Yao H, He G, Song L, Liu N, Wang Y, et al. Effects of Lovastatin on MDA-MB-231 Breast Cancer Cells: An Antibody Microarray Analysis. J Cancer. 2016;7:192-9.

25. Yao H, He G, Yan S, Chen C, Song L, Rosol TJ, et al. Triple-negative breast cancer: is there a treatment on the horizon? Oncotarget. 2017;8:1913-24.

26. Zhang Z, Tan M, Xie Z, Dai L, Chen Y, Zhao Y. Identification of lysine succinylation as a new posttranslational modification. Nat Chem Biol. 2011;7:58-63.

27. Wang C, Zhang C, Li X, Shen J, Xu Y, Shi H, et al. CPT1A-mediated succinylation of S100A10 increases human gastric cancer invasion. J Cell Mol Med. 2019;23:293-305.

28. Song Y, Wang J, Cheng Z, Gao P, Sun J, Chen X, et al. Quantitative global proteome and lysine succinylome analyses provide insights into metabolic regulation and lymph node metastasis in gastric cancer. Sci Rep. 2017;7:42053.

29. Gao X, Bao H, Liu L, Zhu W, Zhang L, Yue L. Systematic analysis of lysine acetylome and succinylome reveals the correlation between modification of H2A.X complexes and DNA damage response in breast cancer. Oncol Rep. 2020;43:1819-30.

30. Chen XF, Tian MX, Sun RQ, Zhang ML, Zhou LS, Jin L, et al. SIRT5 inhibits peroxisomal ACOX1 to prevent oxidative damage and is downregulated in liver cancer. EMBO Rep. 2018;19.

31. Yang X, Wang Z, Li X, Liu B, Liu M, Liu L, et al. SHMT2 Desuccinylation by SIRT5 Drives Cancer Cell Proliferation. Cancer Res. 2018;78:372-86.

32. Li QQ, Hao J-J, Zhang Z, Krane LS, Hammerich KH, Sanford T, et al. Proteomic analysis of proteome and histone post-translational modifications in heat shock protein 90 inhibition-mediated bladder cancer therapeutics. Scientific Reports. 2017;7:201.

33. Li Y, Zhang T, Korkaya H, Liu S, Lee HF, Newman B, et al. Sulforaphane, a dietary component of broccoli/broccoli sprouts, inhibits breast cancer stem cells. Clin Cancer Res. 2010;16:2580-90.

34. Peng Y, He G, Tang D, Xiong L, Wen Y, Miao X, et al. Lovastatin Inhibits Cancer Stem Cells and Sensitizes to Chemo- and Photodynamic Therapy in Nasopharyngeal Carcinoma. J Cancer. 2017;8:1655-64.

35. Chiu HW, Fang WH, Chen YL, Wu MD, Yuan GF, Ho SY, et al. Monascuspiloin enhances the radiation sensitivity of human prostate cancer cells by stimulating endoplasmic reticulum stress and inducing autophagy. PLoS One. 2012;7:e40462. 
36. Li H, He G, Yao H, Song L, Zeng L, Peng X, et al. TGF-beta Induces Degradation of PTHrP Through Ubiquitin-Proteasome System in Hepatocellular Carcinoma. J Cancer. 2015;6:511-8.

37. Lee S. Post-translational modification of proteins in toxicological research: focus on lysine acylation. Toxicol Res. 2013;29:81-6.

38. Zhang J, Tian XJ, Zhang H, Teng Y, Li R, Bai F, et al. TGF-beta-induced epithelial-to-mesenchymal transition proceeds through stepwise activation of multiple feedback loops. Sci Signal. 2014;7:ra91.

39. Sotiropoulou PA, Christodoulou MS, Silvani A, Herold-Mende C, Passarella D. Chemical approaches to targeting drug resistance in cancer stem cells. Drug Discov Today. 2014;19:1547-62.

40. Yilmaz M, Christofori G. EMT, the cytoskeleton, and cancer cell invasion. Cancer Metastasis Rev. 2009;28:15-33.

41. Kalluri R, Weinberg RA. The basics of epithelial-mesenchymal transition. J Clin Invest. 2009;119:1420-8.

42. Shankar J, Nabi IR. Actin cytoskeleton regulation of epithelial mesenchymal transition in metastatic cancer cells. PLoS One. 2015;10:e0119954.

43. Peng JM, Bera R, Chiou CY, Yu MC, Chen TC, Chen CW, et al. Actin cytoskeleton remodeling drives epithelial-mesenchymal transition for hepatoma invasion and metastasis in mice. Hepatology. 2018;67:2226-43.

44. Sousa-Squiavinato ACM, Rocha MR, Barcellos-de-Souza P, de Souza WF, Morgado-Diaz JA. Cofilin-1 signaling mediates epithelial-mesenchymal transition by promoting actin cytoskeleton reorganization and cell-cell adhesion regulation in colorectal cancer cells. Biochim Biophys Acta Mol Cell Res. 2019;1866:418-29.

45. Pollard TD, Cooper JA. Actin, a central player in cell shape and movement. Science. 2009;326:120812.

46. Schmoller KM, Lieleg O, Bausch AR. Structural and viscoelastic properties of actin/filamin networks: cross-linked versus bundled networks. Biophys J. 2009;97:83-9.

47. Maninova M, Caslavsky J, Vomastek T. The assembly and function of perinuclear actin cap in migrating cells. Protoplasma. 2017;254:1207-18.

\section{Figures}


A

\section{Cellular Component}

T1 vs C1 T2 vs C2 T1 vs T2 C1 vs C2

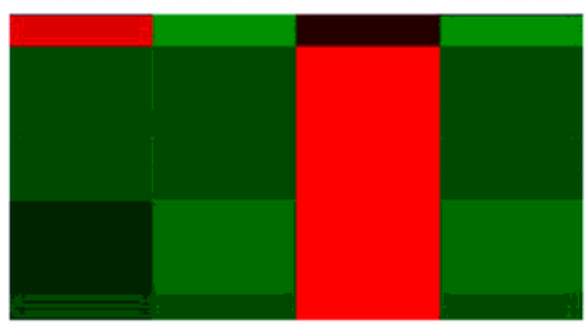

actin cytoskeleton

endosomal part

endosome membrane

vacuolar part

bounding membrane of organelle

membrane part

cell-substrate adherens junction

cell-substrate junction

focal adhesion

cell junction

\section{Molecular Function}

T1 vs C1 T2 vs C2 T1 vs T2 C1 vs C2

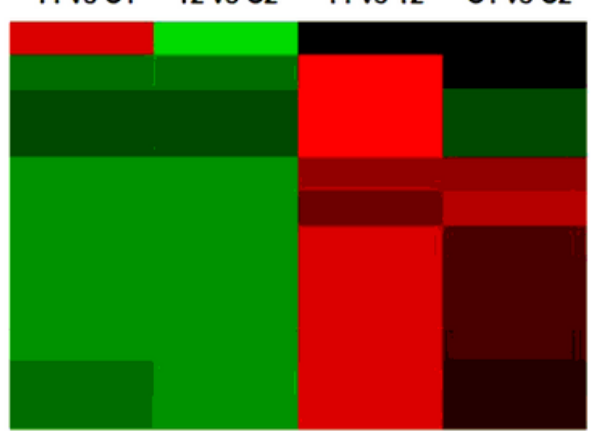

actin binding

protein homodimerization activity protein dimerization activity identical protein binding

calcium ion binding

protein binding involved in cell adhesion

glycoprotein binding

drug binding

metal ion binding

cation binding

cadherin binding

cell adhesion molecule binding
T1: MDA-MB-231, LV-treated Zscore(-log IO(Fisher 's exact test P value))

C1: MDA-MB-231, Control

T2: MDA-MB-453, LV-treated

C2: MDA-MB-453, Control

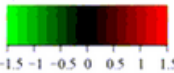

B
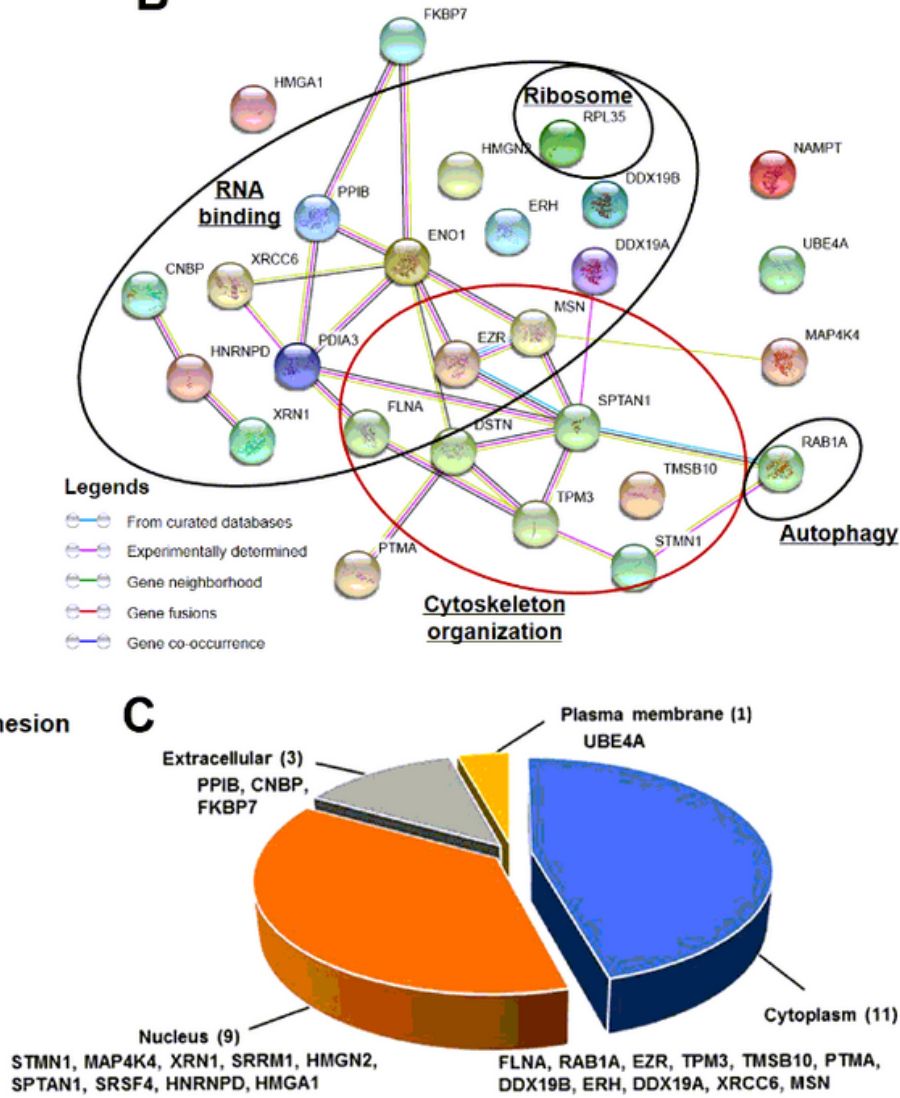

\section{Figure 1}

Lovastatin induces lysine succinylation (Ksucc) of cytoskeleton-associated proteins. a Gene Ontology (GO) analysis showing the enrichment of Ksucc-modified proteins involved in cytoskeleton organization in MDA-MB-231 CSCs treated with lovastatin $(1 \mu \mathrm{M}, 48 \mathrm{~h})$. b Protein-protein interaction network generated using the STRING database (https://string-db.org/) showing the functional groups of lovastatindysregulated Ksucc-modified proteins in MDA-MB-231 CSCs. c Pie-chart showing the distribution of Ksucc-modified proteins in different cellular components of MDA-MB-231 CSCs. LV, lovastatin. 
A

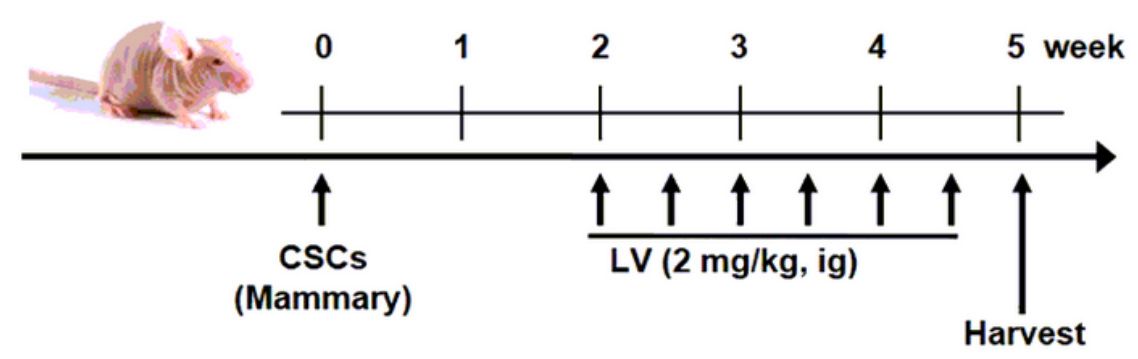

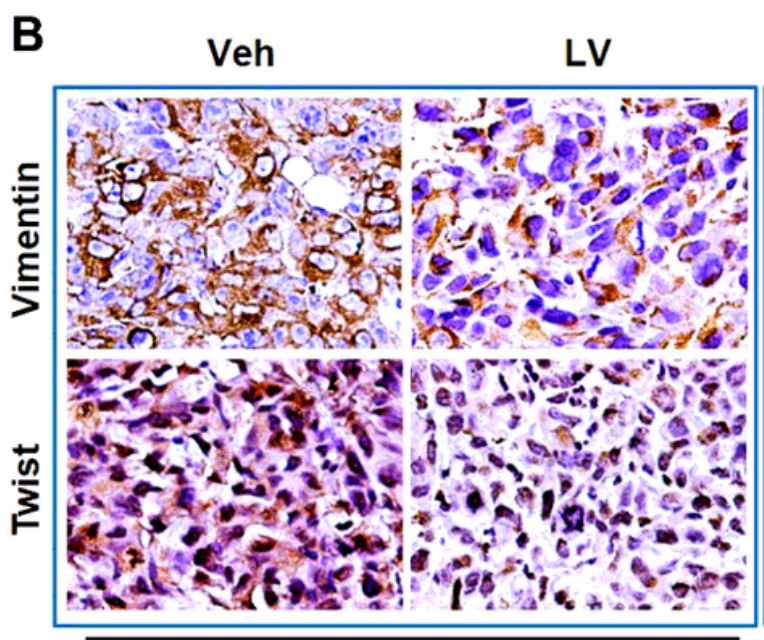

MDA-MB-231 CSCs

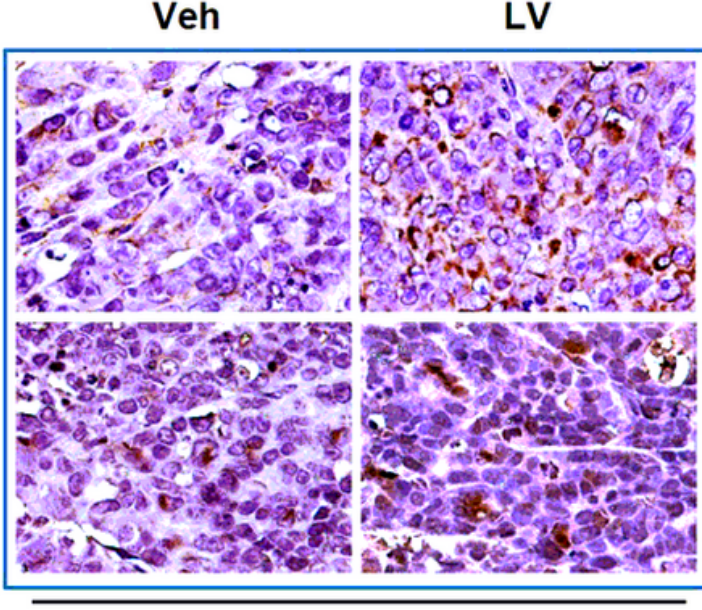

MDA-MB-453 CSCs
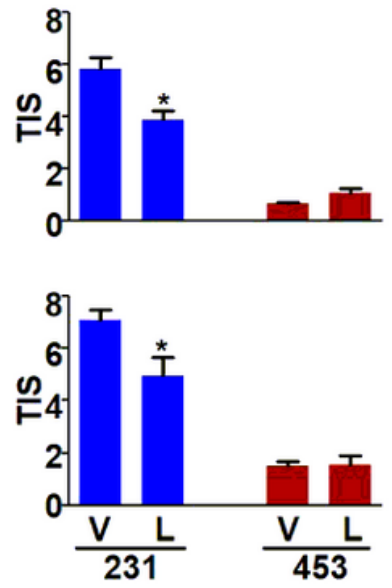

Figure 2

Lovastatin inhibits EMT of TNBC CSCs in vivo. a Schematic diagram showing the experimental procedure of mouse model of EMT phenotype. b Representative images of immunohistochemical staining for Vimentin and Twist in orthotopic tumors derived from MDA-MB-231 and MDA-MB-453 CSCs. The nucleus was counterstained by hematoxylin. Right, quantification of the total intensity score (TIS). ${ }^{*} \mathrm{P}<0.05$, compared with control; $V$ or Veh, vehicle; L or LV, lovastatin; ig: intragastric administration. 


\section{A}

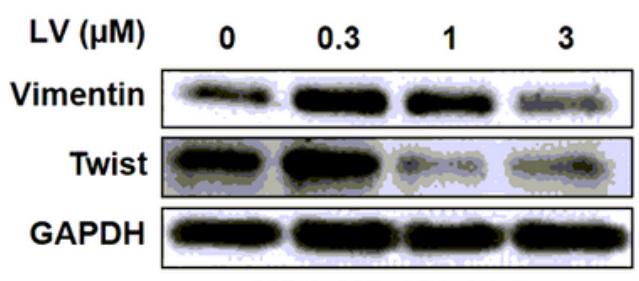

MDA-MB-231 CSCs
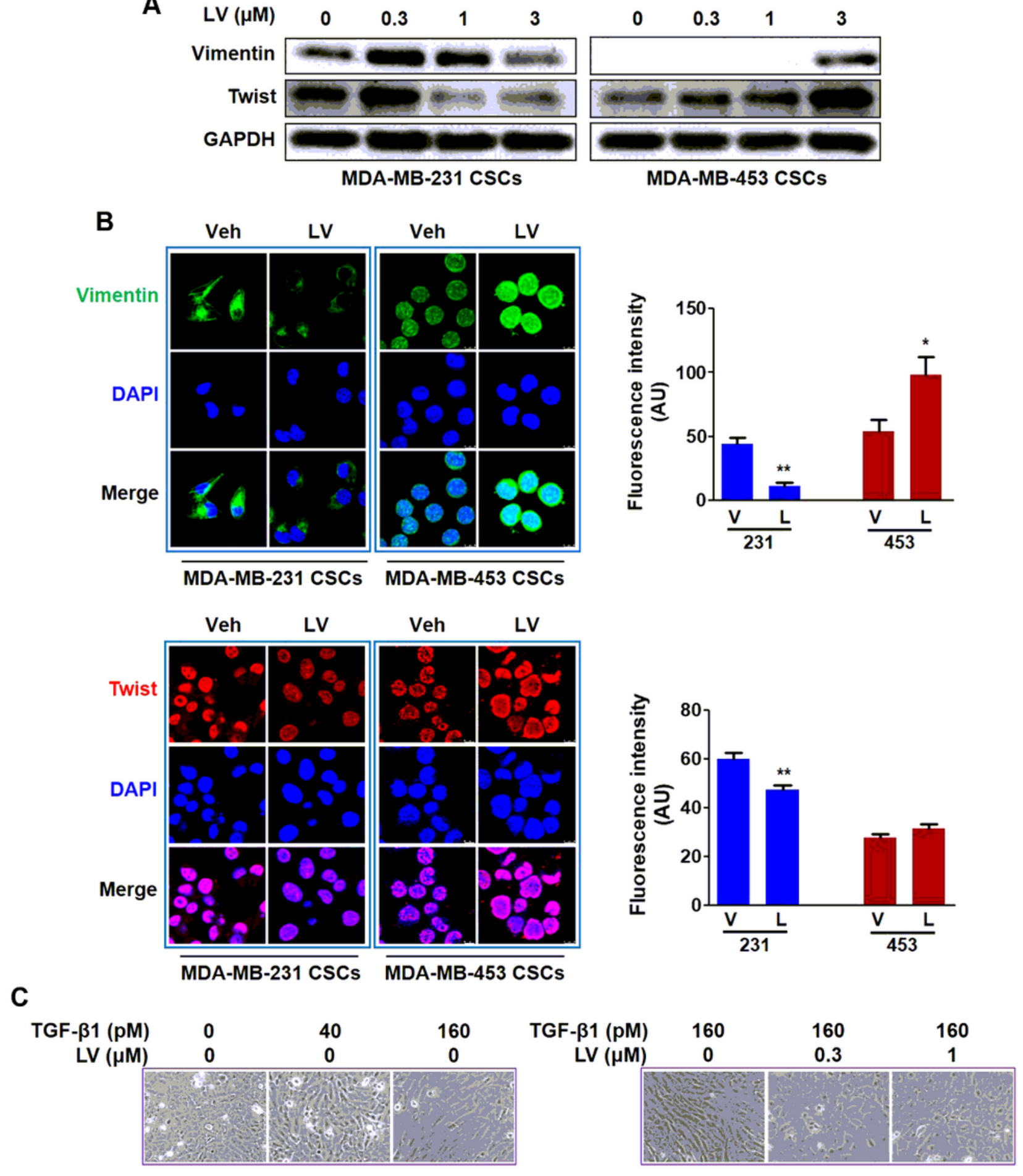

MDA-MB-453 CSCs

\section{Figure 3}

Lovastatin inhibits EMT of TNBC CSCs in vitro. a MDA-MB-231 CSCs and MDA-MB-453 CSCs were treated with lovastatin or vehicle for $48 \mathrm{~h}$ and the protein levels of Vimentin and Twist were analyzed by western blot analysis. b Representative confocal images immunofluorescence staining of Vimentin (green) and Twist (red) on MDA-MB-231 CSCs treated in a similar way as in Fig. 3a. Nuclei were stained with 4', 6-diamidino-2-phenylindole (DAPI) (blue). Original magnification: 63x. Right, quantification of 
immunofluorescence intensity. c MCF10A cells were cultured for 7 days with or without different concentrations of TGF- $\beta 1$ and/or lovastatin, morphological changes were observed by microscopic examination. ${ }^{*} \mathrm{P}<0.05$, ${ }^{*} \mathrm{P}<0.01$, compared with control; $\mathrm{V}$ or Veh, vehicle; L or LV, lovastatin; $\mathrm{AU}$, arbitrary unit.
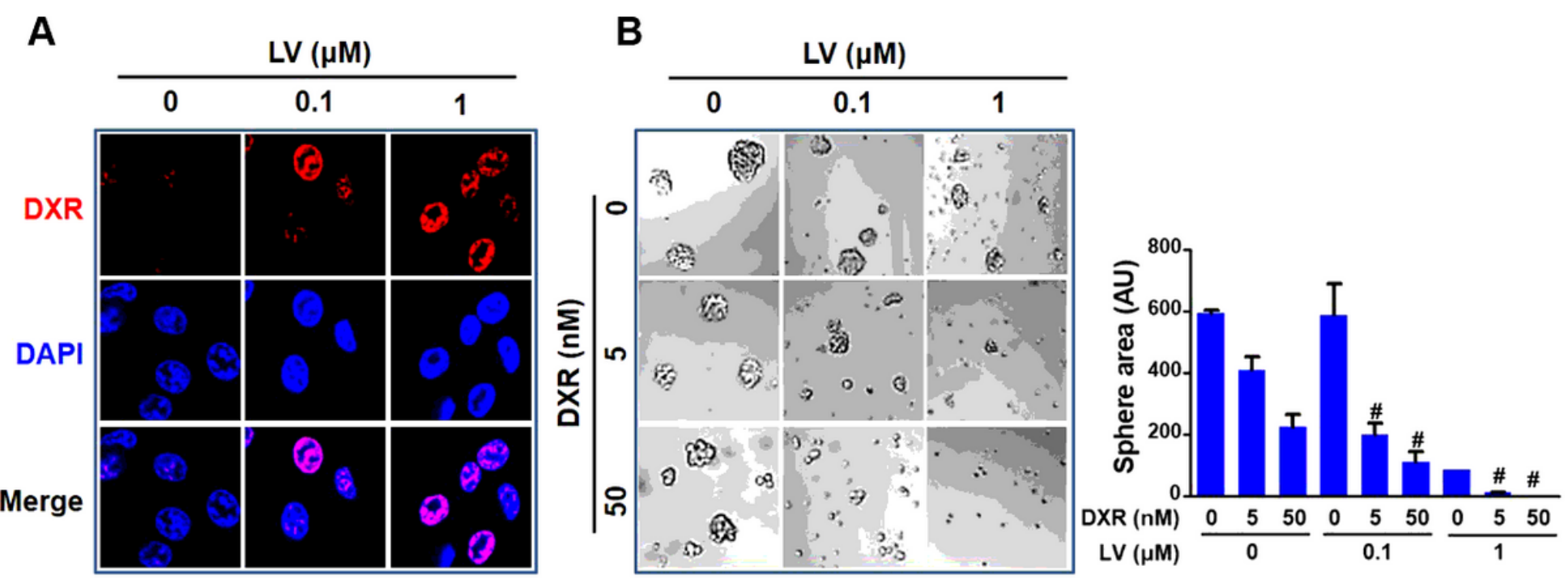

Figure 4

Lovastatin increases the sensitivity of TNBC CSCs to doxorubicin. a Intracellular accumulation of DXR promoted by lovastatin. MDA-MB-231 CSCs were treated for $24 \mathrm{~h}$ with DXR $(5 \mu \mathrm{M})$, alone or in combination with lovastatin, and the intracellular fluorescence of DXR (red) was observed by laser scanning confocal microscopy. Original magnification: 63x. b Synergistic effect between lovastatin and DXR on inhibiting tumorsphere-forming activity. MDA-MB-231 CSCs were cultured in the presence or absence of lovastatin and/or DXR and the tumorspheres were observed and recorded $5 \mathrm{~d}$ after treatment. Right, quantifications of the areas of tumorspheres. $\# \mathrm{Cl}<1.0$, showing synergism between the two drugs. LV, lovastatin; DXR, doxorubicin; AU, arbitrary unit. 
A

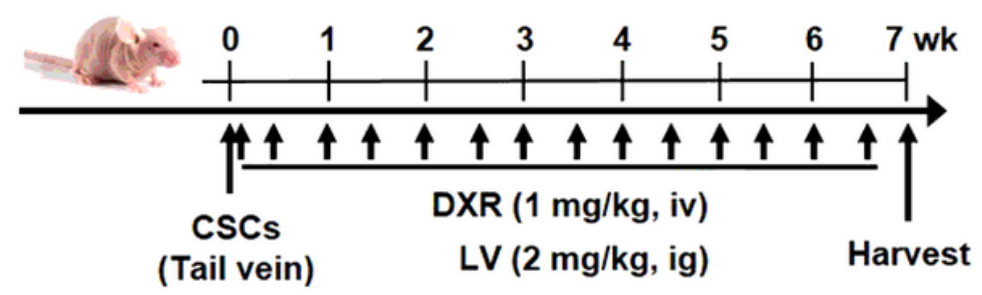

B

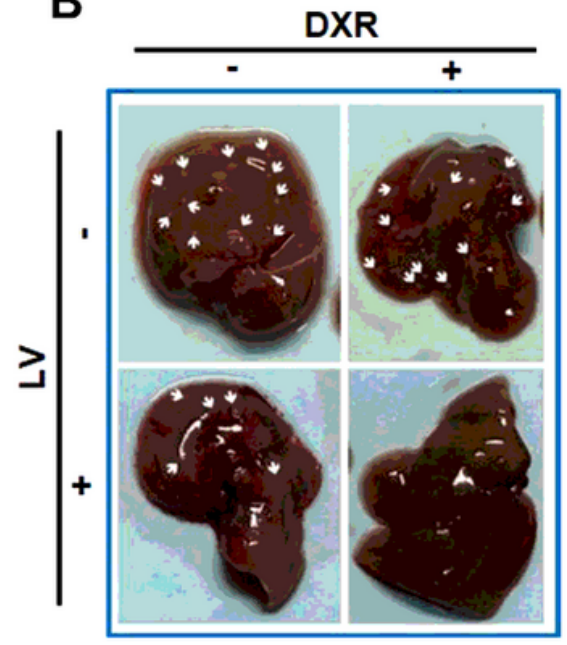

C

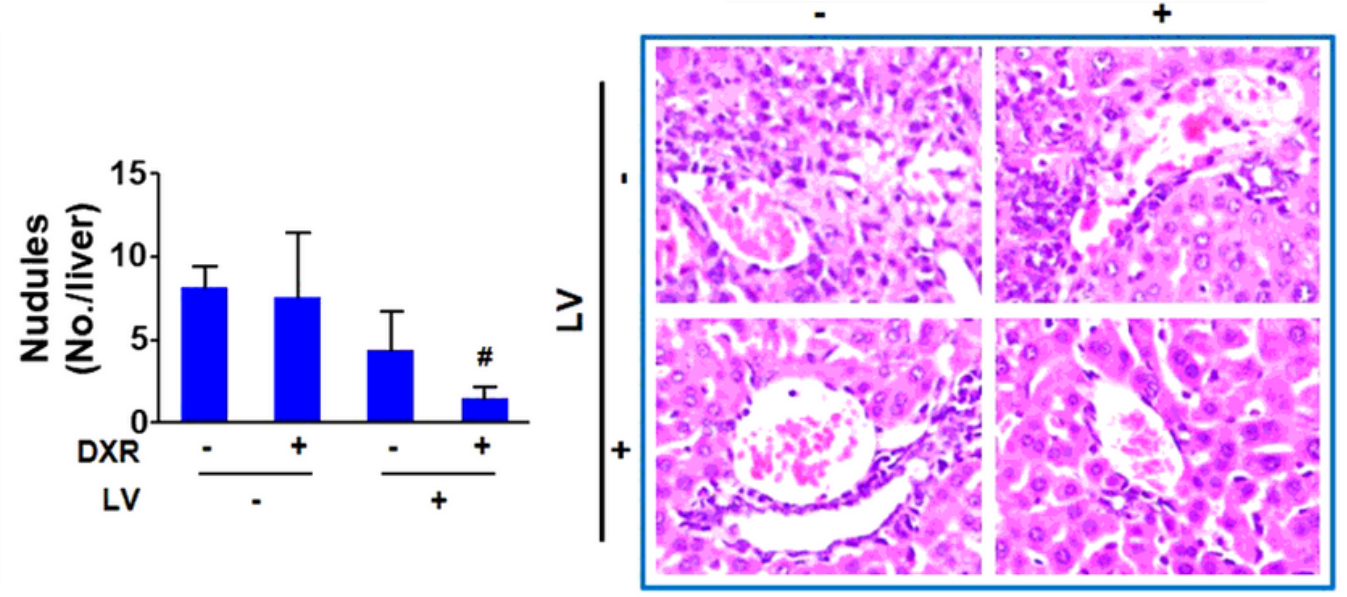

\section{Figure 5}

Lovastatin inhibits metastasis of TNBC CSCs in vivo. a Schematic of the mouse model of tumor metastasis. $b$ Representative images of the livers from each group of mice. Arrows indicate the tumor nodules on the liver. Right, quantifications of the tumor nodules on liver surface. c Representative H\&Estained histopathological images showing reduced colonization of tumor cells in the liver by combination treatment with lovastatin and DXR. Original magnification: $40 \times . \# \mathrm{Cl}<1.0$, showing synergism between the two drugs. LV, lovastatin; DXR, doxorubicin; iv: intravenous injection; ig: intragastric administration. 
A
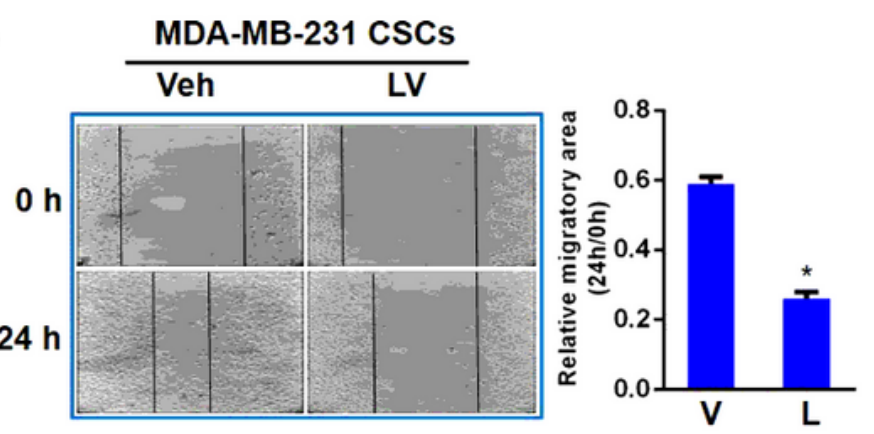
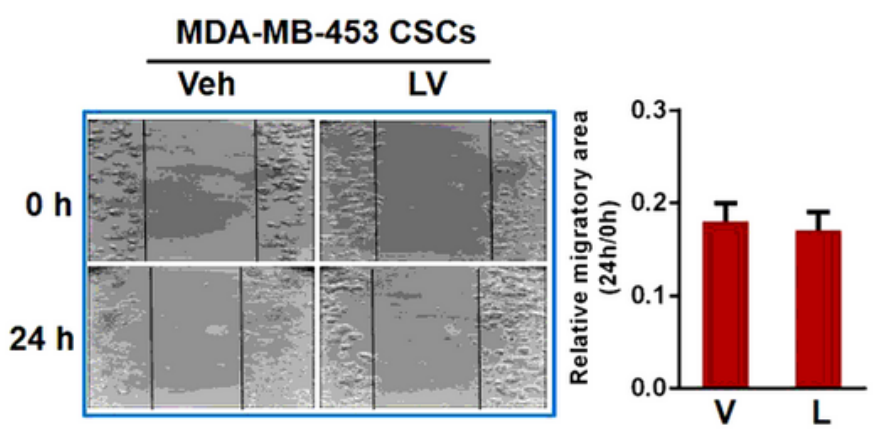

B
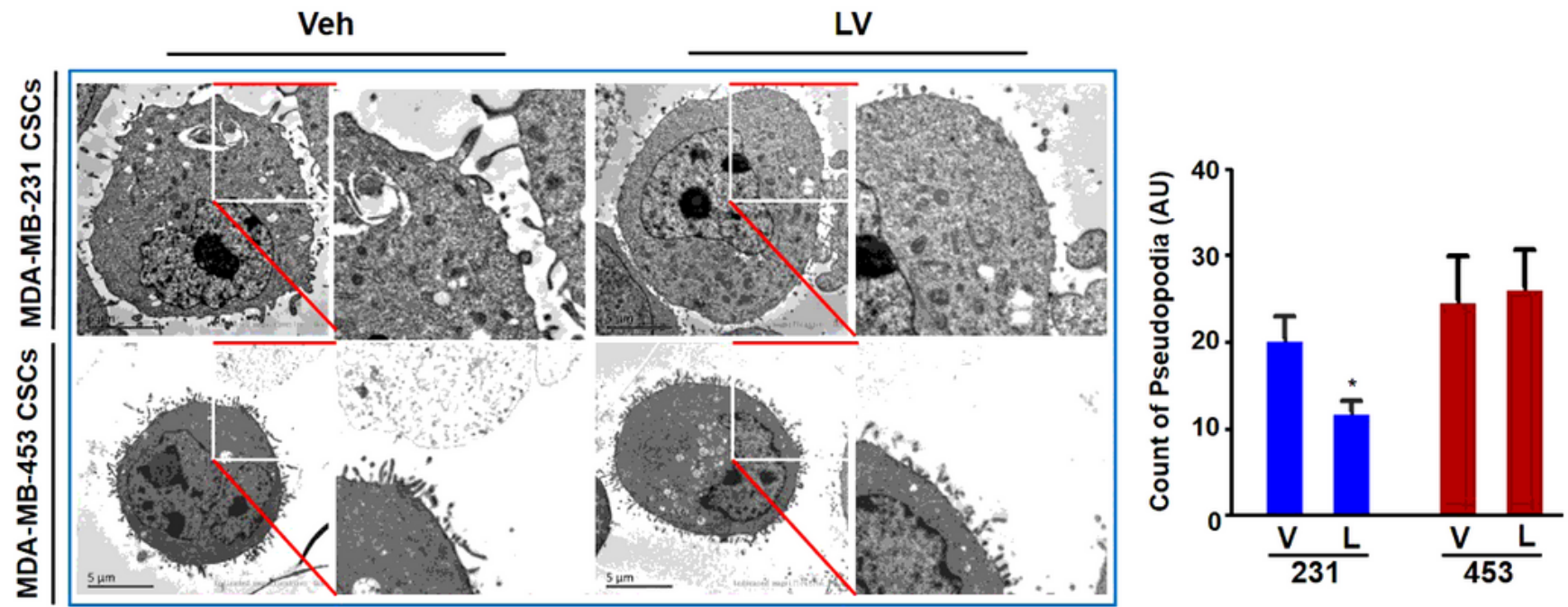

C

F-actin

DAPI

Merge

Enlarge
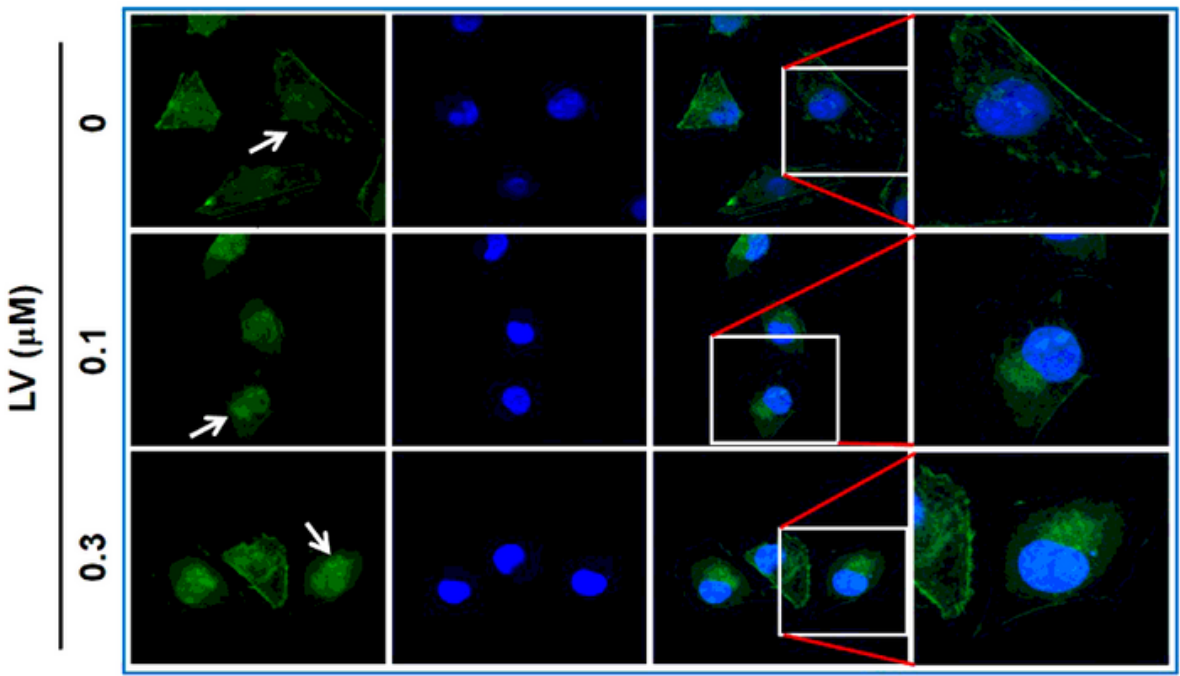

\section{Figure 6}

Lovastatin inhibits metastasis of TNBC CSCs in vitro. a Representative cell images of wound-healing assay in MDA-MB-231 and MDA-MB-453 CSCs treated with vehicle or lovastatin ( $1 \mu \mathrm{M}, 24 \mathrm{~h})$. Right, quantification of relative migratory area. $b$ Representative TEM micrographs showing the pseudopodia in MDA-MB-231 and MDA-MB-453 CSCs after treatment with lovastatin $(1 \mu \mathrm{M}, 48 \mathrm{~h})$. Scale bar $=5 \mu \mathrm{m}$. C Representative confocal images of immunofluorescence staining for F-actin in MDA-MB-231 after 
treatment with vehicle or lovastatin $(0.1$ or $0.3 \mu \mathrm{M}, 48 \mathrm{~h})$. Blue, DAPI staining of the nucleus. Original magnification: $63 \times . \# \mathrm{Cl}<1.0$, showing synergism between the two drugs. ${ }^{*} \mathrm{P}<0.05$, compared with control; $\mathrm{V}$ or Veh, vehicle; L or LV, lovastatin; AU, arbitrary
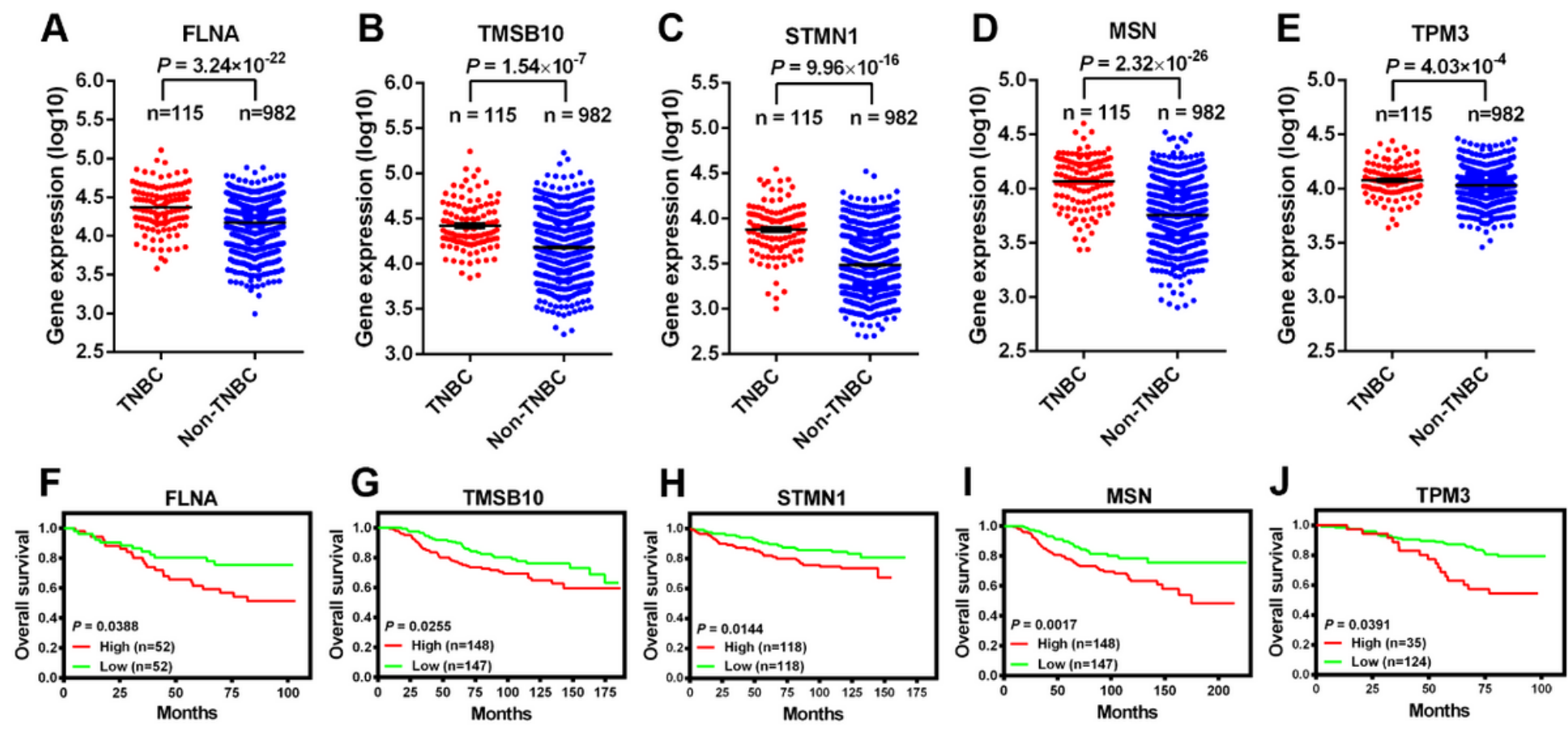

Figure 7

Higher expression levels of cytoskeleton-associated genes are characteristic of TNBC and predict poorer overall survival in breast cancer patients. a-e The expression of FLNA (a), TMSB10 (b), STMN1 (c), MSN (d), SPTAN1 (e) analyzed between TNBC and non-TNBC clinical samples from The Cancer Genome Atlas (TCGA), which was contained in the online cBioPortal for cancer genomics database. $f-j$ The overall survival in breast cancer patients retrieved from the online database (PROGgeneV2) between high and low expression of FLNA (f, dataset GSE42568), TMSB10 (g, dataset NKI), STMN1 (h, dataset GSE3494_U133A ), MSN (i, dataset NKI), TPM3 (j, dataset GSE1456-GPL97).

\section{Supplementary Files}

This is a list of supplementary files associated with this preprint. Click to download.

- EMTSupplementaryfile20210103.docx 